\title{
ESTIMATED USE \\ OF GROUND WATER \\ FOR IRRIGATION IN WISCONSIN, 1984
}

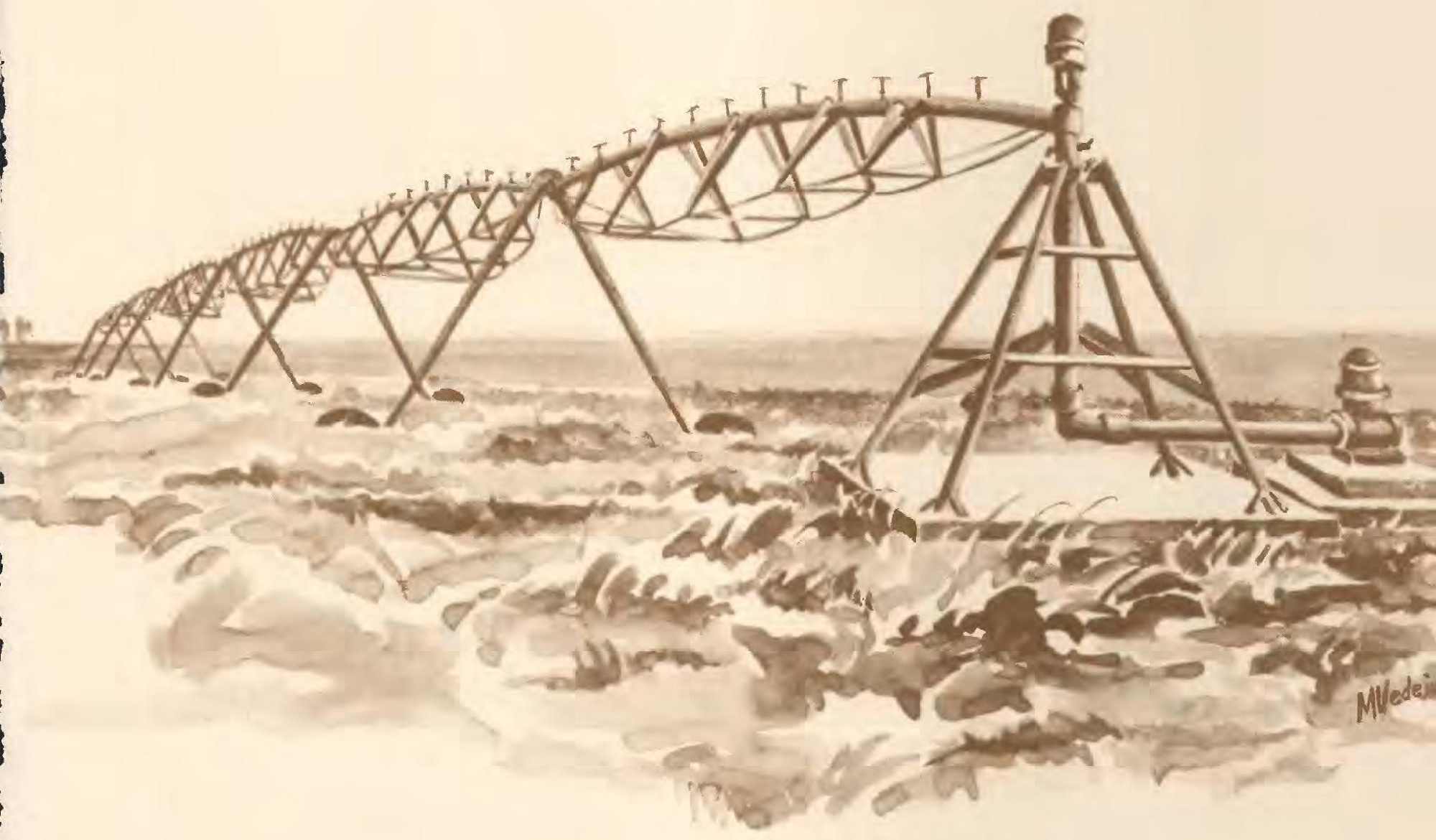

Prepared by United States Department of the Interior, Geological Survey 


\section{ESTIMATED USE \\ OF GROUND WATER \\ FOR IRRIGATION IN WISCONSIN, 1984}

By

J. T. Krohelski, B. R. Ellefson, and C. A. Storlie

Water-Resources Investigations 86-4079

Prepared by

United States Department of the Interior

Geological Survey

In cooperation with the

University of Wisconsin-Extension,

Agricultural Engineering Department

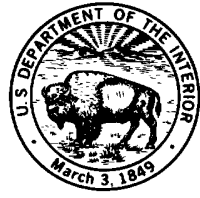

Madison, Wisconsin

1987 


\section{UNITED STATES DEPARTMENT OF THE INTERIOR DONALD PAUL HODEL, Secretary}

GEOLOGICAL SURVEY

Dallas L. Peck, Director

For additional information write to:

District Chief

U.S. Geological Survey, WRD

6417 Normandy Lane

Madison, Wisconsin 53719
Copies of this report can be purchas?d from:

Open-File Services Section Western Distribution Branch

U.S. Geological Survey

Box 25425, Federal Center

Denver, Colorado 80225

(Telephone: (303) 236-7476) 


\section{CONTENTS}

Page

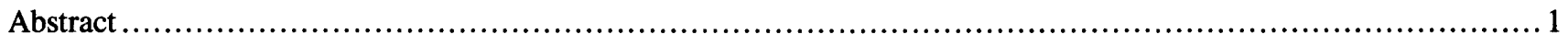

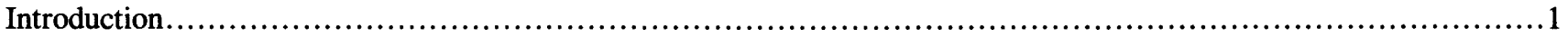

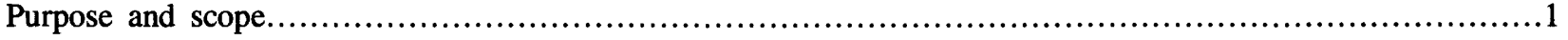

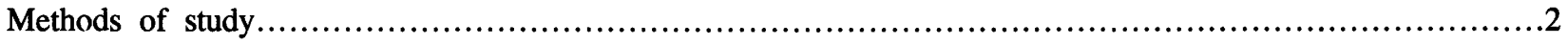

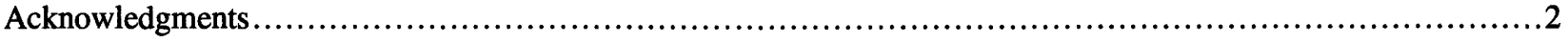

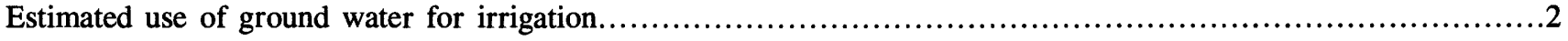

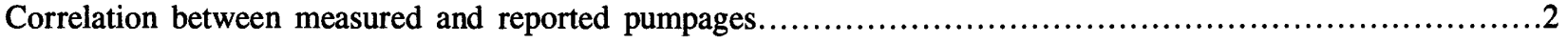

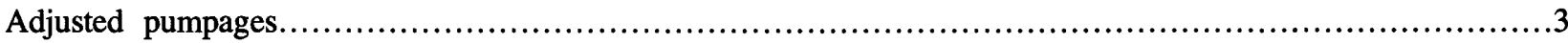

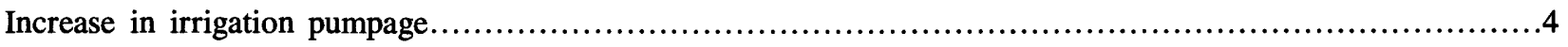

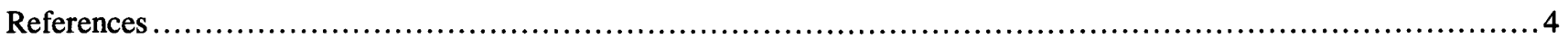

\section{ILLUSTRATIONS}

Plate 1. Map showing location of irrigation wells and major irrigation areas in Wisconsin (in pocket)

Figure 1. Map showing location of irrigation wells measured for this study .3

\section{TABLES}

Table 1. Location, irrigation area, system type, and pumpage for measured irrigation wells.......................5

2. Distribution of 1984 ground-water pumpage for irrigation in Wisconsin,

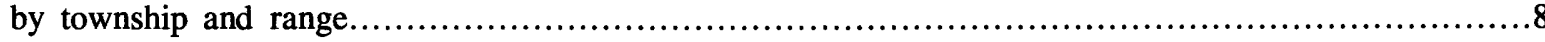

3. Distribution of 1984 ground-water pumpage for irrigation in Wisconsin, by county....................12 


\section{FACTORS FOR CONVERTING INCH-POUND UNITS \\ TO INTERNATIONAL SYSTEM (SI) UNITS}

For the use of readers who prefer the International System of Units (SI), the conversion factors for the terms used in this report are listed below.

Multiply inch-pound units

gallon (gal)

gallon per minute $(\mathrm{gal} / \mathrm{min})$

million gallons per year

(Mgal/yr)
By

0.003785

0.003785

0.003785
To obtain SI unit

cubic meters $\left(\mathrm{m}^{3}\right)$

cubic meter per minute $\left(\mathrm{m}^{3} / \mathrm{min}\right)$

million cubic maters per year $\left(\mathrm{Mm}^{3} / \mathrm{yr}\right)$ 


\title{
ESTIMATED USE \\ OF GROUND WATER \\ FOR IRRIGATION IN WISCONSIN, 1984
}

\author{
By \\ J. T. Krohelski, B. R. Ellefson, and C. A. Storlie
}

\begin{abstract}
The flow from a sample of 143 irrigation wells from 6 irrigation areas in Wisconsin was measured using an external flowmeter during the 1983-84 irrigation seasons. The measured pumpages were correlated with reported pumpages. The equation that describes this correlation is:

$$
Q_{m}=0.86 \times Q_{r}
$$

where $\quad \mathrm{Q}_{m}$ is the measured pumpage and

$Q_{r}$ is the reported pumpage.

This equation was used to adjust all reported irrigation pumpage, yielding a more accurate estimate of total irrigation water use. The maximum total irrigation pumpage for 1984 was estimated to be 31,700 million gallons by using the equation and accounting for both reported and unreported pumpage.

From 1979 to 1984, irrigation pumpage increased a maximum of 25 percent and the maximum number of irrigation wells increased 32 percent.
\end{abstract}

\section{INTRODUCTION}

Irrigation is used in Wisconsin to increase the quantity and quality of crop yields, to protect against crop loss during dry periods, and to maintain the quality of recreational facilities. Sloggett (1985) estimated that irrigation increased 72 percent in Wisconsin between 1974 and 1983, based on an increase in the State's irrigated acreage. The largest increase in irrigation was during 1976 and 1977 (based on irrigation-well permit applications). This increase was undoubtedly due to a drought beginning in 1976 (Tom
Calabresa, Wisconsin Department of Natural Resource , oral commun., 1985). After 1977 the installation rate of rew irrigation systems declined. Ground water accounts for approximately 97 percent of the water used for irrigation in Wisconsin (Lawrence and Ellefson, 1982, p. 98).

An accurate assessment of current irrigation practices largely depends on the analyses of an accurate data base. Irrigators using $70 \mathrm{gal} / \mathrm{min}$ or more from a well or a combination of wells on one property are required to report pumpage to the Wisconsin Department of Natural Resources (DNR). In addition to these data, the Soil Conservation Service and the University of Wisconsin-Extension have compiled data on system type, water sources, power so urces, and irrigated soil types (Soil Conservation Service, 1977).

Determining the accuracy of reported pumpages is useful to both irrigators and scientists. It is becoming increaringly important to know how much water is being applied to $\varepsilon$ field. Water applied in excess of plant requirements can promote leaching of fertilizer and pesticide from the soil to the groundwater reservoir and increase the cost of pumping. Storlie (Agricultural Engineering, University of WisconsinMadison, written commun., 1985) reports that many Wisconsin irrigators overwater their crops, some by as much as 100 percent of the crop demand.

\section{Purpose and Scope}

The purpose of this report is to present a more accurate estimate of statewide ground-water use for irrigation than previously made. Irrigators generally calculate (and report) their pumpage based on rated pump capacity and the number of hours the system was running. Pumpage estimates can be incorrect if pump efficiency, pump capacity, or total pumpage hours are in error. 
In this study pumpage from a statistical sample of irrigation wells was measured, correlated with reported pumpage, and the relationship was applied to statewide reported pumpage data. The report does not include information on irrigation from sources other than ground water, or nonagricultural irrigation. Reported pumpage data were assembled by DNR during the spring of 1985 . These data are entered into a computer file that is maintained by DNR.

\section{Methods of Study}

The flow rate from irrigation wells was measured using a ClampItron Model 240 flowmeter ${ }^{1}$. This flowmeter uses ultrasonic waves to measure flow and is both noninvasive and nondestructive. Transducers are mounted on opposite sides of an irrigation pipe. A signal is passed through the pipe and fluid, and returned to the electronic assembly where it is translated into a flow rate. The ClampItron meter was calibrated at known flow rates ranging from 100 to 1,200 $\mathrm{gal} / \mathrm{min}$. An accuracy of about plus or minus 5 percent was obtained at flow rates less than $130 \mathrm{gal} / \mathrm{min}$. At flow rates greater than $300 \mathrm{gal} / \mathrm{min}$ an accuracy of plus or minus 3 percent was obtained. Storlie $(1985$, p. 31$)$ presents a complete description of the flowmeter.

The State was divided into six irrigation areas to ease data collection and interpretation (fig. 1). The five major areas are groups of counties that have the highest concentration of irrigators. Irrigation areas include: the northwest, northeast, central sands, lower sands, south, and "other" (which contains irrigation wells not located in the five major areas). It was determined that 230 wells should be measured to obtain a Statewide statistical sample having a confidence interval of plus or minus 10 percent (the number 230 was derived from a regression analysis using 33 measured wells). A random-number generator was used to select approximately 14 percent of the irrigation wells from each irrigation area, for a total of 350 wells. This number allowed wells that could not be measured for various reasons (such as a temporarily unused well or wells for which permission to measure could not be obtained) to be dropped from the sample.

About half of the irrigation wells in the State are located in the central sands irrigation area. Most measured wells are in the central sands irrigation area because the area contains a large number of wells and because it is readily accessible from Madison. Measurements in more distant areas were difficult to obtain because unpredictable storms would shut down irrigation.

Measurements of flow from 143 irrigation systems were made during the 1983 and 1984 irrigation seasons. Regression techniques were used to determine the correlation between measured flow and reported flow at the measured sites.

1 The use of the brand name in this report is for identification purpose only and does not imply endorsement by the U.S. Geological Survey.
Attempts were made to correlate irrigatior area and type of irrigation system to measured flow. Although the age of the irrigation-system components may be a factor in the accuracy of reported pumpages, it was not conside"ed in this study.

Statewide irrigation-water use estimated in this report has been incorporated into the State Water-Use Data System (SWUDS). SWUDS was created in 1984 as part of a national effort to quantify water use and is maintained by the U.S. Geological Survey. SWUDS is updated annually and will provide data for estimating annual water use.

\section{Acknowledgments}

Appreciation is expressed to the many irrigators who allowed measurement of their wells. DNR provided a listing of reported pumpages used in this report. Appreciation is also given to Rick Stowell, an agricultural engineering student, who measured wells during the 1984 irrigation season, and to Kraig Rury, for writing the computer programs that aided in analyzing and plotting data.

\section{ESTIMATED USE OF GROUND WATER FOR IRRIGAT ON}

Estimates of total ground-water pumpage were improved by using the relationship between measured and reported pumpage.

\section{Correlation Between Measured and Reported Pumpares}

The discharge from 143 irrigation we'ls was measured. Table 1 shows the location, irrigation area, system type, reported pumpage, and measured pumpage for each of these wells. There is a strong correlation between measured and reported pumpages (STD ERROR $=130.1$ ). The equation that describes this correlation is:

$$
Q_{m}=0.86 \times Q_{r}
$$

where $Q_{m}$ is the measured pumpage and

$Q_{r}$ is the reported pumpage.

Statistical analysis also indicates a difference in the correlation of pumpage between central-pivot and traveling-gunirrigation systems. Where data are availab'e on system type, accuracy of reported pumpages may be slightly improved by applying one of the following equations (STD ERROR = 125.): 


$$
Q_{m}=0.84 \times Q_{c}
$$

where: $Q_{m}$ is measured pumpage for a central-pivot system,

$Q_{c}$ is the reported pumpage for a central-pivot system, and

$$
Q_{m}=0.98 \times Q_{t}
$$

where $Q_{m}$ is measured pumpage for a traveling-gun system and

$Q_{t} \quad$ is the reported pumpage for a traveling-gun system.

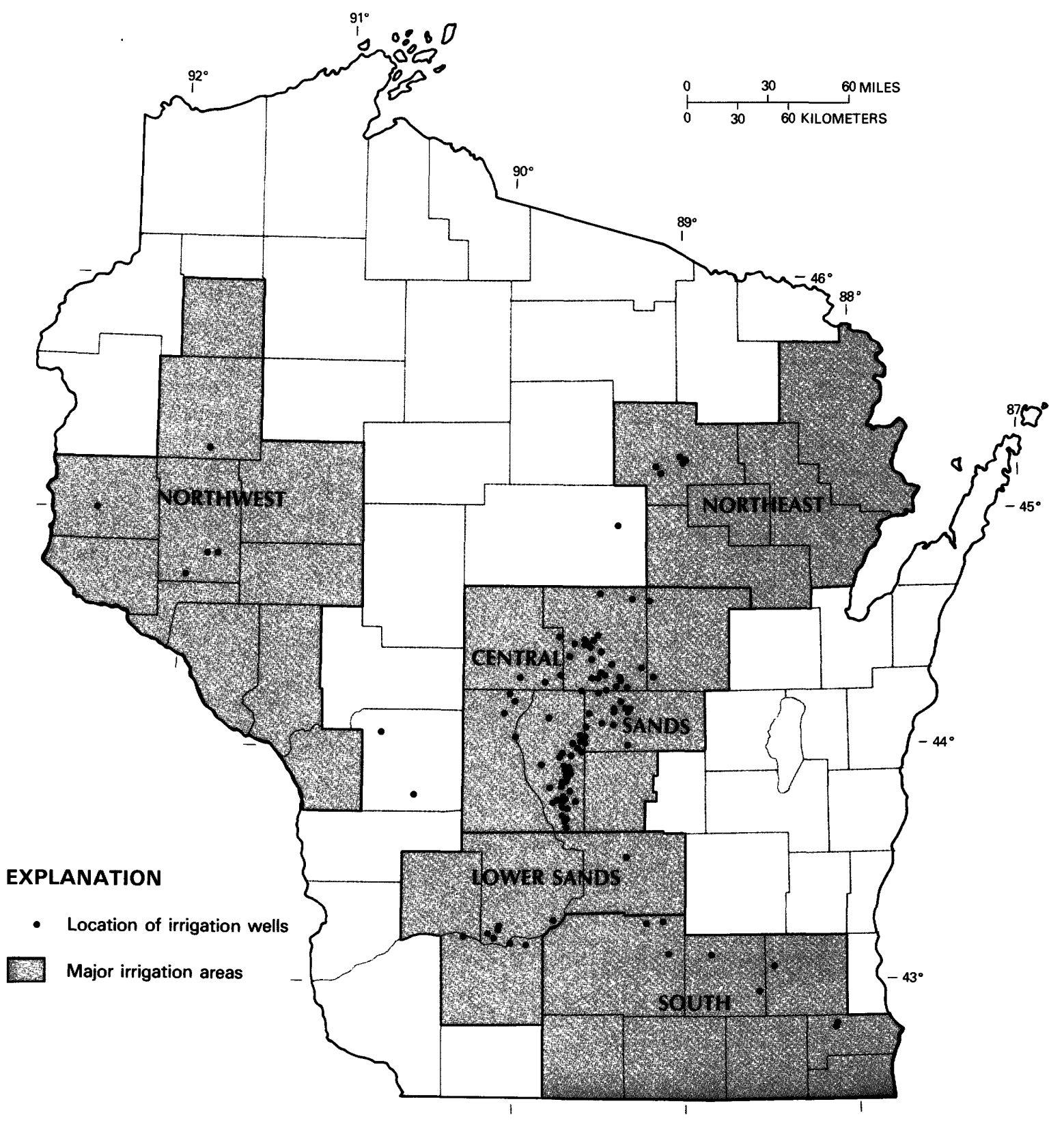

Figure 1. Location of irrigation wells measured for this study.
An attempt was made to correlate reported pump'ge to measured pumpage within each of the irrigation areas. Because of the lack of data in most irrigation areas, the validity of the regression equations is questionable and they are not presented in this report.

\section{Adjusted Pumpages}

Equation 1 was used to adjust 1984 reported pumpages. Table 2 shows reported irrigation pumpage and adjusted pumpage by township and range. Table 3 summarizes these data by county. Note that the adjusted total pumpage is lower than the reported pumpage. 
The adjusted total pumpage shown in tables 2 and 3 is a minimum value $(18,182 \mathrm{Mgal} / \mathrm{yr})$ because 43 percent of the irrigators did not report pumpage to DNR. Only those wells in the DNR file of high-capacity irrigation wells that had a completion date and monthly pumpage values are shown in tables 2 and 3, and on plate 1. Wells that either did not have a completion date or did not have pumpage values were excluded.

A maximum total pumpage for the State was calculated by including estimates for these wells without reported data. The average adjusted pumpage per irrigation well with reported data is $11.6 \mathrm{Mgal} / \mathrm{yr}$. Multiplying $11.6 \mathrm{Mgal} / \mathrm{yr}$ times 1,162 (the number of irrigation wells without reported data) equals $13,500 \mathrm{Mgal} / \mathrm{yr}$; the estimated additional pumpage from wells without reported data. This value, added to the adjusted total pumpage from tables 2 and 3, should approximate the maximum total pumpage. Based on these procedures the minimum total irrigation pumpage for 1984 was 18,182 and the maximum was $31,700 \mathrm{Mgal} / \mathrm{yr}$.

\section{Increase in Irrigation Pumpage}

Irrigation pumpage in Wisconsin increased by 25 percent between 1979 and 1984. In 1979 Lawrence and Ellef- son (1982, p. 98) reported that $29,600 \mathrm{Mgal}$ were pumped for irrigation, based on the DNR file. Using equation 1 to adjust the 1979 pumpage so that it is comprrable to adjusted 1984 pumpage yields 25,337 Mgal in 1979.

In 1979 and 1984 there were 2,065 and 2,733 irrigation wells, respectively (the totals include both wells with reported and unreported pumpage data). This represents a 32-percent increase in the number of irrigation wells for the 5-year period from 1979 to 1984.

\section{REFERENCES}

Lawrence, C. L., and Ellefson, B. R., 19², Water use in Wisconsin, 1979: U.S. Geological Survey WaterResources Investigations 82-444, 98 p.

Soil Conservation Service and Universit ${ }^{\prime}$ of WisconsinExtension, 1977, 1977 Irrigation Da+a Survey.

Sloggett, G., 1985, Draft of Energy and U.S. Agriculture: Irrigation pumping, 1974-83: U.S. Department of Agriculture, Economic Research Service.

Storlie, C. A., 1985, Irrigation pumping plant and water use evaluation: Madison, University of WisconsinAgricultural Engineering master's th $\odot$ sis, 163 p. 
Table 1. Location, irrigation area, system type, and pumpage for measured irrigation wells

\begin{tabular}{|c|c|c|c|c|c|c|}
\hline County & $\begin{array}{l}\text { Locati } \\
\text { Township }\end{array}$ & $\begin{array}{l}\text { ion } \\
\text { Range }\end{array}$ & $\begin{array}{c}\text { Measured } \\
(\mathrm{gal} / \mathrm{min})\end{array}$ & $\begin{array}{l}\text { Reported } \\
\text { (gal/min) }\end{array}$ & Irrigation area & Type of system \\
\hline Adams & $14 \mathrm{~N}$ & $06 \mathrm{E}$ & 560 & 368 & Central Sands & Center pivot \\
\hline Adams & $14 \mathrm{~N}$ & $06 E$ & 509 & 550 & Central Sands & Center pivot \\
\hline Adams & $14 \mathrm{~N}$ & 07E & 672 & 700 & Central Sands & Center pivot \\
\hline Adams & $14 \mathrm{~N}$ & O7E & 608 & 850 & Central Sands & Center pivot \\
\hline Adams & $15 \mathrm{~N}$ & $06 \mathrm{E}$ & 701 & 950 & Central Sands & Center pivot \\
\hline Adams & $15 \mathrm{~N}$ & $06 \mathrm{E}$ & 486 & 600 & Central Sands & Traveling gun \\
\hline Adams & $15 \mathrm{~N}$ & $06 \mathrm{E}$ & 705 & 800 & Central Sands & Center pivot \\
\hline Adams & $15 \mathrm{~N}$ & $06 E$ & 551 & 650 & Central Sands & Center pivot \\
\hline Adams & $15 N$ & $06 \mathrm{E}$ & 531 & 700 & Central Sands & Center pivot \\
\hline Adams & $15 \mathrm{~N}$ & $06 \mathrm{E}$ & 548 & 700 & Central Sands & Center pivot \\
\hline Adams & $15 \mathrm{~N}$ & $06 \mathrm{E}$ & 695 & 725 & Central Sands & Center pivot \\
\hline Adams & $15 \mathrm{~N}$ & $06 E$ & 486 & 750 & Central Sands & Center pivot \\
\hline Adams & $15 \mathrm{~N}$ & 07E & 650 & 1,000 & Central Sands & Center pivot \\
\hline Adams & $15 \mathrm{~N}$ & $07 E$ & 519 & 1.000 & Central Sands & Center pivot \\
\hline Adams & $15 \mathrm{~N}$ & O7E & 775 & 775 & Central Sands & Center pivot \\
\hline Adams & $15 \mathrm{~N}$ & $07 E$ & 515 & 1,000 & Central Sands & Center pivot \\
\hline Adams & $15 \mathrm{~N}$ & 07E & 775 & 1,000 & Central Sands & Center pivot \\
\hline Adams & $15 \mathrm{~N}$ & $07 E$ & 800 & 1,000 & Central Sands & Center pivot \\
\hline Adams & $15 \mathrm{~N}$ & 07E & 450 & 500 & Central Sands & Center pivot \\
\hline Adams & $16 \mathrm{~N}$ & $06 E$ & 789 & 1,000 & Central Sands & Center pivot \\
\hline Adams & $16 \mathrm{~N}$ & $06 \mathrm{E}$ & 672 & 700 & Central Sands & Center pivot \\
\hline Adams & $16 \mathrm{~N}$ & $06 E$ & 946 & 800 & Central Sands & Center pivot \\
\hline Adams & $16 \mathrm{~N}$ & $07 E$ & 780 & 1,000 & Central Sands & Center pivot \\
\hline Adams & $16 \mathrm{~N}$ & $07 \mathrm{E}$ & 822 & 1,000 & Central Sands & Center pivot \\
\hline Adams & $16 \mathrm{~N}$ & 07E & 780 & 1,000 & Central Sands & Center pivot \\
\hline Adams & $16 \mathrm{~N}$ & $07 E$ & 793 & 1,000 & Central Sands & Center pivot \\
\hline Adams & $16 \mathrm{~N}$ & 07E & 788 & 1,000 & Central Sands & Center pivot \\
\hline Adams & $17 \mathrm{~N}$ & $06 E$ & 660 & 700 & Central Sands & Center pivot \\
\hline Adams & $17 \mathrm{~N}$ & $06 E$ & 1,036 & 1,100 & Central Sands & Center pivot \\
\hline Adams & $17 \mathrm{~N}$ & 07E & 660 & 1,000 & Central Sands & Center pivot \\
\hline Adams & $17 \mathrm{~N}$ & $07 E$ & 619 & 650 & Central Sands & Center pivot \\
\hline Adams & $17 \mathrm{~N}$ & $07 E$ & 936 & 1,000 & Central Sands & Center pivot \\
\hline Adams & $17 \mathrm{~N}$ & 07E & 598 & 900 & Central Sands & Center pivot \\
\hline Adams & $17 \mathrm{~N}$ & $07 E$ & 810 & 900 & Central Sands & Center pivot \\
\hline Adams & $17 \mathrm{~N}$ & $07 \mathrm{E}$ & 707 & 900 & Central Sands & Center pivot \\
\hline Adams & $17 \mathrm{~N}$ & 07E & 548 & 1,000 & Central Sands & Center pivot \\
\hline Adams & $17 \mathrm{~N}$ & O7E & 614 & 800 & Central Sands & Center pivot \\
\hline Adams & $17 \mathrm{~N}$ & O7E & 598 & 800 & Central Sands & Center pivot \\
\hline Adams & $17 \mathrm{~N}$ & $07 E$ & 964 & 900 & Central Sands & Center pivot \\
\hline Adams & $17 N$ & 07E & 740 & 850 & Central Sands & Center pivot \\
\hline Adams & $17 \mathrm{~N}$ & $07 E$ & 872 & 1,000 & Central Sands & Center pivot \\
\hline Adams & $17 \mathrm{~N}$ & $07 \mathrm{E}$ & 747 & 850 & Central Sands & Center pivot \\
\hline Adams & $17 \mathrm{~N}$ & 07E & 766 & 900 & Central Sands & Center pivot \\
\hline Adams & $18 \mathrm{~N}$ & 07E & 728 & 800 & Central Sands & Center pivot \\
\hline Adams & $18 \mathrm{~N}$ & $07 E$ & 423 & 450 & Central Sands & Center pivot \\
\hline Adams & $18 \mathrm{~N}$ & 07E & 901 & 900 & Central Sands & Center pivot \\
\hline Adams & $18 \mathrm{~N}$ & 07E & 872 & 900 & Central Sands & Center pivot \\
\hline Adams & $18 \mathrm{~N}$ & $07 E$ & 942 & 1,000 & Central Sands & Center pivot \\
\hline Adams & $18 \mathrm{~N}$ & $07 \mathrm{E}$ & 295 & 650 & Central Sands & Center pivot \\
\hline Adams & $18 \mathrm{~N}$ & O7E & 800 & 875 & Central Sands & Center pivot \\
\hline
\end{tabular}


Table 1. Location, irrigation area, system type, and pumpage for measured irrigation wells-Continued

\begin{tabular}{|c|c|c|c|c|c|c|}
\hline County & $\begin{array}{l}\text { Locatic } \\
\text { Township }\end{array}$ & $\begin{array}{l}\text { on } \\
\text { Range }\end{array}$ & $\begin{array}{c}\text { Measured } \\
\text { (gal/min) }\end{array}$ & $\begin{array}{l}\text { Reported } \\
\text { (gal/min) }\end{array}$ & Irrigation area & Type of system \\
\hline Adams & $18 \mathrm{~N}$ & 07E & 722 & 1,100 & Central Sands & Certer pivot \\
\hline Adams & $18 \mathrm{~N}$ & $07 \mathrm{E}$ & 506 & 500 & Central Sands & Center pivot \\
\hline Adams & $19 N$ & 06E & 568 & 700 & Central Sands & Center pivot \\
\hline Adams & $20 \mathrm{~N}$ & $07 \mathrm{E}$ & 1,197 & 1,100 & Central Sands & Center pivot \\
\hline Barron & $32 \mathrm{~N}$ & $12 W$ & 640 & 800 & North West & Center pivot \\
\hline Barron & $34 N$ & $11 W$ & 651 & 800 & North West & Traveling gun \\
\hline Columbia & $12 \mathrm{~N}$ & $10 E$ & 520 & 600 & Central Sands & Center pivot \\
\hline Dane & $05 \mathrm{~N}$ & $09 \mathrm{E}$ & 592 & 775 & Southern & Center pivot \\
\hline Dane & $08 \mathrm{~N}$ & $12 E$ & 419 & 450 & Southern & Traveling gun \\
\hline Dane & $09 \mathrm{~N}$ & $11 \mathrm{E}$ & 678 & 900 & Southern & Center pivot \\
\hline Dane & $09 \mathrm{~N}$ & $11 \mathrm{E}$ & 661 & 800 & Southern & Center pivot \\
\hline Dunn & $26 \mathrm{~N}$ & $13 W$ & 728 & 1,000 & North West & Center pivot \\
\hline Dunn & $27 \mathrm{~N}$ & $11 W$ & 756 & 1,000 & North West & Center pivot \\
\hline Dunn & $27 \mathrm{~N}$ & $11 W$ & 636 & 1,000 & North West & Center pivot \\
\hline Dunn & $27 N$ & $12 W$ & 680 & 700 & North West & Center pivot \\
\hline Iowa & $08 \mathrm{~N}$ & 02E & 790 & 900 & Lower Sands & Center pivot \\
\hline Iowa & $08 \mathrm{~N}$ & $03 \mathrm{E}$ & 505 & 525 & Lower Sands & Traveling gun \\
\hline I owa & $08 \mathrm{~N}$ & $05 \mathrm{E}$ & 455 & 500 & Lower Sands & Traveling gun \\
\hline Jefferson & $06 \mathrm{~N}$ & $16 \mathrm{E}$ & 1,978 & 1,750 & other & Traveling gun \\
\hline Jefferson & $08 \mathrm{~N}$ & $14 \mathrm{E}$ & 323 & 450 & other & Tra"eling gun \\
\hline Juneau & $19 \mathrm{~N}$ & $04 \mathrm{E}$ & 445 & 750 & Central Sands & Center pivot \\
\hline Juneau & $20 \mathrm{~N}$ & $04 \mathrm{E}$ & 630 & 800 & Central Sands & Center pivot \\
\hline Juneau & $20 \mathrm{~N}$ & $04 \mathrm{E}$ & 670 & 1,000 & Central Sands & Center pivot \\
\hline Juneau & $20 N$ & $04 \mathrm{E}$ & 774 & 1,000 & Central Sands & Center pivot \\
\hline Kenosha & $01 \mathrm{~N}$ & $19 E$ & 350 & 520 & other & Hant-moved \\
\hline Langlade & $31 N$ & $11 E$ & 614 & 650 & North East & Center pivot \\
\hline Langlade & $31 \mathrm{~N}$ & $11 \mathrm{E}$ & 825 & 800 & North East & Certer pivot \\
\hline Langlade & $32 N$ & $12 E$ & 1,010 & 975 & North East & Center pivot \\
\hline Langlade & $32 \mathrm{~N}$ & $12 E$ & 382 & 400 & North East & Traveling gun \\
\hline Langlade & $32 N$ & $12 E$ & 382 & 400 & North East & Traveling gun \\
\hline Monroe & $18 \mathrm{~N}$ & 04E & 479 & 450 & other & Traveling gun \\
\hline Portage & $21 \mathrm{~N}$ & $08 \mathrm{E}$ & 299 & 500 & Central Sands & Center pivot \\
\hline Portage & $21 \mathrm{~N}$ & $08 \mathrm{E}$ & 893 & 950 & Central Sands & Certer pivot \\
\hline Portage & $21 \mathrm{~N}$ & $08 \mathrm{E}$ & 544 & 800 & Central Sands & Center pivot \\
\hline Portage & $21 \mathrm{~N}$ & $08 \mathrm{E}$ & 778 & 800 & Central Sands & Certer pivot \\
\hline Portage & $21 \mathrm{~N}$ & $09 \mathrm{E}$ & 596 & 700 & Central Sands & Center pivot \\
\hline Portage & $21 \mathrm{~N}$ & $09 \mathrm{E}$ & 565 & 600 & Central Sands & Center pivot \\
\hline Portage & $21 \mathrm{~N}$ & $09 \mathrm{E}$ & 868 & 800 & Central Sands & Center pivot \\
\hline Portage & $21 \mathrm{~N}$ & $09 E$ & 680 & 775 & Central Sands & Center pivot \\
\hline Portage & $21 \mathrm{~N}$ & $09 E$ & 507 & 600 & Central Sands & Center pivot \\
\hline Por tage & $21 \mathrm{~N}$ & $10 E$ & 697 & 800 & Central Sands & Center pivot \\
\hline Portage & $22 \mathrm{~N}$ & $07 \mathrm{E}$ & 522 & 475 & Central Sands & Traveling gun \\
\hline Portage & $22 \mathrm{~N}$ & $07 \mathrm{E}$ & 624 & 800 & Central Sands & Center pivot \\
\hline Portage & $22 \mathrm{~N}$ & $08 \mathrm{E}$ & 561 & 900 & Central Sands & Center pivot \\
\hline Portage & $22 \mathrm{~N}$ & $08 \mathrm{E}$ & 842 & 900 & Central Sands & Center pivot \\
\hline Portage & $22 \mathrm{~N}$ & $08 \mathrm{E}$ & 680 & 800 & Central Sands & Center pivot \\
\hline Portage & $22 \mathrm{~N}$ & $08 \mathrm{E}$ & 551 & 1,000 & Central Sands & Center pivot \\
\hline Portage & $23 N$ & O7E & 1,175 & 1,250 & Central Sands & Center pivot \\
\hline Portage & $23 N$ & 08E & 806 & 900 & Central Sands & Center pivot \\
\hline Portage & $23 \mathrm{~N}$ & $08 \mathrm{E}$ & 664 & 900 & Central Sands & Center pivot \\
\hline
\end{tabular}


Table 1. Location, irrigation area, system type, and pumpage for measured irrigation wells-Continued

\begin{tabular}{|c|c|c|c|c|c|c|}
\hline County & $\begin{array}{l}\text { Locatio } \\
\text { Township }\end{array}$ & $\begin{array}{l}\text { on } \\
\text { Range }\end{array}$ & $\begin{array}{c}\text { Measured } \\
(\text { gal/min) }\end{array}$ & $\begin{array}{l}\text { Reported } \\
\text { (gal//min) }\end{array}$ & Irrigation area & Type of system \\
\hline Portage & $23 N$ & $08 \mathrm{E}$ & 708 & 708 & Central Sands & Center pivot \\
\hline Portage & $23 N$ & $08 \mathrm{E}$ & 398 & 550 & Central Sands & Traveling gun \\
\hline Portage & $23 N$ & $08 \mathrm{E}$ & 583 & 700 & Central Sands & Center pivot \\
\hline Portage & $23 N$ & 08E & 970 & 1,000 & Central Sands & Center pivot \\
\hline Portage & $23 N$ & $08 \mathrm{E}$ & 892 & 775 & Central Sands & Center pivot \\
\hline Portage & $23 N$ & $08 \mathrm{E}$ & 980 & 950 & Central Sands & Center pivot \\
\hline Portage & $23 N$ & $08 \mathrm{E}$ & 883 & 800 & Central Sands & Center pivot \\
\hline Portage & $23 N$ & $08 \mathrm{E}$ & 582 & 800 & Central Sands & Center pivot \\
\hline Portage & $25 N$ & O9E & 863 & 1,000 & Central Sands & Center pivot \\
\hline Portage & $25 \mathrm{~N}$ & $10 \mathrm{E}$ & 693 & 750 & Central Sands & Center pivot \\
\hline Portage & $25 N$ & $10 \mathrm{E}$ & 551 & 560 & Central Sands & Traveling gun \\
\hline Racine & $04 \mathrm{~N}$ & $20 \mathrm{E}$ & 701 & 700 & other & Traveling gun \\
\hline Racine & $04 N$ & $20 \mathrm{E}$ & 429 & 450 & other & Traveling gur \\
\hline Sauk & $08 \mathrm{~N}$ & O3E & 1,016 & 1,100 & Lower Sands & Traveling gun \\
\hline Sauk & $08 N$ & O3E & 908 & 1,000 & Central Sands & Center pivot \\
\hline Sauk & $08 N$ & O3E & 1,176 & 1,000 & Lower Sands & Center pivot \\
\hline Sauk & $09 N$ & O3E & 947 & 1,000 & Central Sands & Center pivot \\
\hline Sauk & $09 \mathrm{~N}$ & $06 \mathrm{E}$ & 1,093 & 1,100 & Lower Sands & Traveling gun \\
\hline st. Croix & $29 N$ & $17 \mathrm{E}$ & 648 & 1,050 & North West & Center pivot \\
\hline Waukesha & $07 \mathrm{~N}$ & $17 \mathrm{E}$ & 623 & 766 & other & Center pivot \\
\hline Waupaca & $21 \mathrm{~N}$ & $11 \mathrm{E}$ & 393 & 550 & Central Sands & Traveling gun \\
\hline Waupaca & $25 \mathrm{~N}$ & $11 \mathrm{E}$ & 764 & 775 & Central Sands & Center pivot \\
\hline Waushara & $18 \mathrm{~N}$ & O8E & 892 & 900 & Central Sands & Center pivot \\
\hline Waushara & $18 N$ & $08 \mathrm{E}$ & 765 & 850 & Central Sands & Center pivot \\
\hline Waushara & $18 \mathrm{~N}$ & $08 \mathrm{E}$ & 872 & 925 & Central Sands & Center pivot \\
\hline Waushara & $18 N$ & $10 E$ & 577 & 900 & Central Sands & Center pivot \\
\hline Waushara & $19 \mathrm{~N}$ & $08 \mathrm{E}$ & 890 & 675 & Central Sands & Center pivot \\
\hline Waushara & $19 \mathrm{~N}$ & $08 \mathrm{E}$ & 553 & 750 & Central Sands & Center pivot \\
\hline Waushara & $19 \mathrm{~N}$ & $08 \mathrm{E}$ & 485 & 625 & Central Sands & Center pivot \\
\hline Waushara & $19 N$ & $08 E$ & 812 & 800 & Central Sands & Center pivot \\
\hline Waushara & $19 N$ & $08 \mathrm{E}$ & 975 & 1,000 & Central Sands & Center pivot \\
\hline Waushara & $19 \mathrm{~N}$ & O9E & 851 & 1,000 & Central Sands & Center pivot \\
\hline Waushara & $19 N$ & 09E & 721 & 750 & Central Sands & Center pivot \\
\hline Waushara & $20 \mathrm{~N}$ & $08 \mathrm{E}$ & 606 & 700 & Central Sands & Center pivot \\
\hline Waushara & $20 N$ & O8E & 784 & 1,000 & Central Sands & Center pivot \\
\hline Waushara & $20 \mathrm{~N}$ & O9E & 740 & 800 & Central Sands & Center pivot \\
\hline Waushara & $20 \mathrm{~N}$ & O9E & 800 & 950 & Central Sands & Center pivot \\
\hline Waushara & $20 N$ & O9E & 362 & 600 & Central Sands & Traveling gun \\
\hline Waushara & $20 N$ & O9E & 575 & 700 & Central Sands & Center pivot \\
\hline Waushara & $20 N$ & O9E & 339 & 420 & Central Sands & Traveling gun \\
\hline Wood & $21 N$ & $05 \mathrm{E}$ & 850 & 900 & Central Sands & Center pivot \\
\hline Wood & $21 N$ & $05 \mathrm{E}$ & 715 & 900 & Central Sands & Center pivot \\
\hline Wood & $21 \mathrm{~N}$ & $06 \mathrm{E}$ & 508 & 800 & Central Sands & Center pivot \\
\hline
\end{tabular}


Table 2. Distribution of 1984 ground-water pumpage for irrigation in Wisconsin, by township and range

\begin{tabular}{|c|c|c|c|c|c|c|c|}
\hline $\begin{array}{l}\text { Town- } \\
\text { ship' }\end{array}$ & Range & $\begin{array}{l}\text { Reported pumpage } \\
\text { (1000's of } \\
\text { gallons) }\end{array}$ & $\begin{array}{l}\text { Adjusted pumpage } \\
\text { (1000's of } \\
\text { gallons) }\end{array}$ & $\begin{array}{l}\text { Town- } \\
\text { ship' }\end{array}$ & Range & $\begin{array}{l}\text { Reported pumpage } \\
\text { (1000's of } \\
\text { gallons) }\end{array}$ & $\begin{array}{l}\text { Adjusted pumpage } \\
\text { (1000's of } \\
\text { gallons) }\end{array}$ \\
\hline 1 & $2 E$ & 0 & 0 & 7 & $9 E$ & 29,216 & 25,008 \\
\hline 1 & $3 \mathrm{E}$ & 0 & 0 & 7 & $13 E$ & 61,607 & 52,735 \\
\hline 1 & $9 \mathrm{E}$ & 76,734 & 65,684 & 7 & $14 \mathrm{E}$ & 0 & 0 \\
\hline 1 & $10 E$ & $\mathbf{9 , 8 8 3}$ & 8,459 & 7 & $16 \mathrm{E}$ & 0 & 0 \\
\hline 1 & $11 E$ & 20,952 & 17,934 & 7 & $17 E$ & 128,524 & 110,016 \\
\hline 1 & $12 \mathrm{E}$ & 49,965 & 42,770 & 7 & $19 \mathrm{E}$ & 3,343 & 2,861 \\
\hline 1 & $13 E$ & 0 & 0 & 7 & $20 E$ & 7,234 & 6,192 \\
\hline 1 & $14 \mathrm{E}$ & 0 & 0 & 8 & $1 \mathrm{~W}$ & 15,624 & 13,374 \\
\hline 1 & $19 \mathrm{E}$ & 1,341 & 1,147 & 8 & $2 W$ & 0 & 0 \\
\hline 1 & $21 E$ & 2,701 & 2,312 & 8 & $1 E$ & 10,368 & 8,875 \\
\hline 2 & $1 E$ & 0 & 0 & 8 & $2 \mathrm{E}$ & 66,679 & 57,077 \\
\hline 2 & 2E & 0 & 0 & 8 & $3 \mathrm{E}$ & 354,125 & 303,130 \\
\hline 2 & $6 E$ & 0 & 0 & 8 & $4 \mathrm{E}$ & 550,820 & 471,501 \\
\hline 2 & $9 \mathrm{E}$ & 56,626 & 48,471 & 8 & $5 \mathrm{E}$ & 81,273 & 69,569 \\
\hline 2 & $10 E$ & 6,912 & 5,916 & 8 & $6 \mathrm{E}$ & 88,396 & 75,667 \\
\hline 2 & $12 \mathrm{E}$ & 7,194 & 6,158 & 8 & $11 \mathrm{E}$ & 791 & 677 \\
\hline 2 & $13 E$ & 86,350 & 73,915 & 8 & $12 E$ & 20,254 & 17,337 \\
\hline 2 & $14 \mathrm{E}$ & 40,401 & 34,583 & 8 & $14 E$ & 4,500 & 3,852 \\
\hline 2 & $20 E$ & 15,776 & 13,504 & 8 & $15 E$ & 0 & 0 \\
\hline 3 & $\mathbf{9 E}$ & 7,396 & 6,331 & 8 & $16 \mathrm{E}$ & 22 & 18 \\
\hline 3 & $10 E$ & 25,200 & 21,571 & 8 & $17 \mathrm{E}$ & 77,760 & 66,562 \\
\hline 3 & $11 \mathrm{E}$ & 50,122 & 42,904 & 8 & $20 \mathrm{E}$ & 20,778 & 17,786 \\
\hline 3 & $12 E$ & 32,454 & 27,780 & 8 & $21 E$ & 15,223 & 13,030 \\
\hline 3 & $13 \mathrm{E}$ & 483 & 413 & 8 & $22 E$ & 22,655 & 19,392 \\
\hline 3 & $14 \mathrm{E}$ & 14,100 & 12,069 & 9 & $2 W$ & 0 & 0 \\
\hline 3 & $15 E$ & 0 & 0 & 9 & $2 \mathrm{E}$ & 14,010 & 11,992 \\
\hline 3 & $16 \mathrm{E}$ & 0 & 0 & 9 & $3 E$ & 187,950 & 160,885 \\
\hline 3 & $17 \mathrm{E}$ & 576 & 493 & 9 & $5 E$ & 11,127 & 9,524 \\
\hline 3 & $20 E$ & 0 & 0 & 9 & $6 E$ & 145,048 & 124,161 \\
\hline 3 & $21 E$ & 19,196 & 16,431 & 9 & $11 E$ & 52,239 & 44,716 \\
\hline 3 & $22 \mathrm{E}$ & 0 & 0 & 9 & $19 \mathrm{E}$ & 0 & 0 \\
\hline 4 & $8 \mathbf{E}$ & 3,415 & 2,923 & 9 & $21 \mathrm{E}$ & 5,586 & 4,781 \\
\hline 4 & $9 \mathrm{E}$ & 0 & 0 & 10 & $5 \mathrm{E}$ & 0 & 0 \\
\hline 4 & $10 \mathrm{E}$ & 14,257 & 12,204 & 10 & $6 E$ & 110,847 & 94,885 \\
\hline 4 & $12 \mathrm{E}$ & 0 & 0 & 10 & $7 \mathrm{E}$ & 0 & 0 \\
\hline 4 & $13 E$ & 0 & 0 & 10 & $10 E$ & 13,950 & 11,941 \\
\hline 4 & $15 \mathrm{E}$ & 0 & 0 & 11 & $8 \mathrm{E}$ & 0 & 0 \\
\hline 4 & $16 E$ & 15,369 & 13,155 & 11 & $10 E$ & 8,424 & 7,210 \\
\hline 4 & $20 E$ & 34,849 & 29,830 & 11 & $12 E$ & 1,416 & 1,212 \\
\hline 5 & $7 \mathrm{E}$ & 85 & 72 & 11 & $13 \mathrm{E}$ & 1,680 & 1,438 \\
\hline 5 & $8 \mathrm{E}$ & 0 & 0 & 11 & $22 \mathrm{E}$ & 540 & 462 \\
\hline 5 & $9 \mathrm{E}$ & 0 & 0 & 12 & $6 E$ & 44,212 & 37,845 \\
\hline 5 & $10 \mathrm{E}$ & 0 & 0 & 12 & $7 E$ & 0 & 0 \\
\hline 5 & $11 E$ & 4,440 & 3,800 & 12 & $9 \mathrm{E}$ & 15,144 & 12,963 \\
\hline 5 & $12 E$ & $\begin{array}{r}4,440 \\
0\end{array}$ & $\begin{array}{r}0,000 \\
0\end{array}$ & 12 & $10 E$ & 17,131 & 14,664 \\
\hline 5 & $14 \mathrm{E}$ & 0 & 0 & 12 & $11 E$ & 2,812 & 2,407 \\
\hline 5 & $15 E$ & 0 & 0 & 12 & $12 \mathrm{E}$ & 360 & 308 \\
\hline 5 & $16 \mathrm{E}$ & 0 & 0 & 12 & $13 \mathrm{E}$ & 4,608 & 3,944 \\
\hline 5 & $20 \mathrm{E}$ & 0 & 0 & 12 & $16 \mathrm{E}$ & 0 & 0 \\
\hline 5 & $21 E$ & 0 & 0 & 12 & $19 \mathrm{E}$ & 0 & 0 \\
\hline 5 & $22 E$ & 0 & 0 & 12 & $22 \mathrm{E}$ & 0 & 0 \\
\hline 6 & $9 \mathrm{E}$ & 1,008 & 862 & 13 & $7 \mathrm{E}$ & 10 & 8 \\
\hline 6 & $11 E$ & 0 & 0 & 13 & $9 \mathrm{E}$ & 0 & 0 \\
\hline 6 & $13 \mathrm{E}$ & 10,305 & 8,821 & 13 & $10 E$ & 0 & 0 \\
\hline 6 & $15 \mathrm{E}$ & 19,800 & 16,948 & 13 & $11 \mathrm{E}$ & 292 & 250 \\
\hline 6 & $16 E$ & 0 & 0 & & & & \\
\hline 6 & $17 E$ & 271 & 232 & \multirow{4}{*}{\multicolumn{4}{|c|}{$\begin{array}{l}\text { Townships with no pumpage are listed because the:' have irrigation wells } \\
\text { without reported pumpage. }\end{array}$}} \\
\hline 6 & $19 \mathrm{E}$ & 0 & 0 & & & & \\
\hline 6 & $21 E$ & 0 & 0 & & & & \\
\hline 7 & $8 \mathrm{E}$ & 0 & 0 & & & & \\
\hline
\end{tabular}


Table 2. Distribution of 1984 ground-water pumpage for irrigation in Wisconsin, by township and range-Continued

\begin{tabular}{|c|c|c|c|c|c|c|c|}
\hline $\begin{array}{l}\text { Town- } \\
\text { ship' }\end{array}$ & Range & $\begin{array}{l}\text { Reported pumpage } \\
\text { (1000's of } \\
\text { gallons) }\end{array}$ & $\begin{array}{l}\text { Adjusted pumpage } \\
\text { (1000's of } \\
\text { gallons) }\end{array}$ & $\begin{array}{l}\text { Town- } \\
\text { ship1 }\end{array}$ & Range & $\begin{array}{l}\text { Reported pumpage } \\
\text { (1000's of } \\
\text { gallons) }\end{array}$ & $\begin{array}{l}\text { Adjusted purrpage } \\
\left(1000^{\prime} \text { 's of }\right. \\
\text { gallons) }\end{array}$ \\
\hline 13 & $12 E$ & 960 & 821 & 18 & $7 E$ & 493,854 & $422,73 ?$ \\
\hline 13 & $13 \mathrm{E}$ & 0 & 0 & 18 & $8 \mathrm{E}$ & 423,148 & 362,214 \\
\hline 14 & $\mathbf{5 E}$ & 7,410 & 6,343 & 18 & $9 \mathrm{E}$ & 228,620 & 195,698 \\
\hline 14 & $6 \mathrm{E}$ & 113,297 & 98,982 & 18 & $10 E$ & 45,794 & $39,19 ?$ \\
\hline 14 & $7 E$ & 114,500 & 98,012 & 18 & $11 E$ & 117,374 & 100,472 \\
\hline 14 & $8 E$ & 0 & 0 & 18 & $15 E$ & 0 & 0 \\
\hline 14 & $9 E$ & 0 & 0 & 18 & $16 \mathrm{E}$ & 4.050 & 3,466 \\
\hline 14 & $10 E$ & 2,152 & 1,842 & 19 & $1 w$ & 1,020 & 873 \\
\hline 14 & $12 E$ & 1,944 & 1,664 & 19 & $3 w$ & 0 & 0 \\
\hline 14 & $13 E$ & 0 & 0 & 19 & $4 W$ & 0 & 0 \\
\hline 14 & $14 \mathrm{E}$ & 0 & 0 & 19 & $5 W$ & 0 & c \\
\hline 14 & $15 \mathrm{E}$ & 0 & 0 & 19 & $9 w$ & 0 & 0 \\
\hline 15 & $7 W$ & 0 & 0 & 19 & 3E & 0 & $c$ \\
\hline 15 & $\mathbf{3 E}$ & 6,023 & 5,155 & 19 & $4 E$ & 145,464 & 124,517 \\
\hline 15 & $5 \mathrm{E}$ & 34,200 & 29,275 & 19 & $5 \mathrm{E}$ & 22,320 & 19,105 \\
\hline 15 & $6 E$ & 290,577 & 248,733 & 19 & $6 E$ & 31,816 & 27,234 \\
\hline 15 & $7 \mathrm{E}$ & 202,937 & 173,714 & 19 & $7 \mathrm{E}$ & 141,722 & 121,314 \\
\hline 15 & $8 E$ & 0 & 0 & 19 & $8 E$ & $1,339,462$ & $1,146,57 ?$ \\
\hline 15 & $9 \mathrm{E}$ & 66,210 & 56,675 & 19 & $9 E$ & 457,161 & 391,329 \\
\hline 15 & $10 E$ & 0 & 0 & 19 & $10 E$ & 344 & 294 \\
\hline 15 & $13 E$ & 6,993 & 5,986 & 19 & $11 E$ & 14,760 & 12,634 \\
\hline 15 & $14 \mathrm{E}$ & 2,700 & 2,311 & 19 & $12 E$ & 1,344 & 1,150 \\
\hline 15 & $17 \mathrm{E}$ & 3,814 & 3,264 & 19 & $19 E$ & 0 & 0 \\
\hline 15 & 23E & 4,133 & 3,537 & 19 & $23 E$ & 5,960 & 5,101 \\
\hline 16 & $6 \mathrm{~W}$ & 0 & 0 & 20 & $4 W$ & 80 & 68 \\
\hline 16 & $7 w$ & 45 & 38 & 20 & $6 w$ & 0 & 0 \\
\hline 16 & $2 \mathrm{E}$ & 2,708 & 2,318 & 20 & $12 W$ & 188,898 & $161,69 \mathrm{R}$ \\
\hline 16 & 3E & 2,811 & 2,406 & 20 & $3 E$ & 0 & 0 \\
\hline 16 & $4 E$ & 0 & 0 & 20 & 4E & 657,036 & 562,422 \\
\hline 16 & $5 E$ & 0 & 0 & 20 & $5 E$ & 154,320 & 132.097 \\
\hline 16 & $6 E$ & 48,359 & 41,395 & 20 & $6 E$ & 117,396 & 100,491 \\
\hline 16 & $7 \mathrm{E}$ & 45,504 & 38,951 & 20 & $7 \mathrm{E}$ & 569,670 & 487,637 \\
\hline 16 & $9 \mathrm{E}$ & 11,427 & 9,781 & 20 & $8 \mathrm{E}$ & $1,560,771$ & $1,336,01 ?$ \\
\hline 16 & $10 E$ & 1,440 & 1,232 & 20 & $9 \mathrm{E}$ & $1,095,721$ & 937,937 \\
\hline 16 & $12 E$ & 0 & 0 & 20 & $10 \mathrm{E}$ & 0 & 6 \\
\hline 16 & $13 E$ & 5,197 & 4,448 & 20 & $11 \mathrm{E}$ & 0 & 0 \\
\hline 16 & $14 \mathrm{E}$ & 9,140 & 7,823 & 20 & $12 E$ & 0 & c \\
\hline 16 & $21 E$ & 8,678 & 7,428 & 20 & $16 E$ & 1,826 & 1,563 \\
\hline 17 & $4 w$ & 4,558 & 3,901 & 20 & $17 E$ & 0 & C \\
\hline 17 & $8 W$ & 0 & 0 & 20 & $18 E$ & 8,097 & 6,931 \\
\hline 17 & $3 E$ & 19,260 & 16,486 & 20 & $19 \mathrm{E}$ & 216 & 184 \\
\hline 17 & $5 E$ & 0 & 0 & 21 & $6 W$ & 0 & 0 \\
\hline 17 & $6 E$ & 84,759 & 72,553 & 21 & $9 \mathrm{~W}$ & 0 & $c$ \\
\hline 17 & $7 \mathrm{E}$ & 352,074 & 301,375 & 21 & $11 W$ & 0 & 0 \\
\hline 17 & $8 \mathbf{E}$ & 0 & 0 & 21 & $12 W$ & 12,000 & $10,27 \hat{a}$ \\
\hline 17 & $9 \mathrm{E}$ & 48,000 & 41,088 & 21 & $4 E$ & 61,080 & 52,284 \\
\hline 17 & $10 E$ & 0 & 0 & 21 & $5 E$ & 159,835 & $136,81 \varepsilon$ \\
\hline 17 & 13E & 26,748 & 22,896 & 21 & $6 E$ & 57,024 & 48,812 \\
\hline 17 & $15 E$ & 0 & 0 & 21 & $7 E$ & 358,400 & $306,79 r$ \\
\hline 18 & $1 W$ & 675 & 577 & 21 & $8 \mathrm{E}$ & 701,180 & 600,210 \\
\hline 18 & $2 W$ & 0 & 0 & 21 & $9 E$ & 617,514 & $528,59 \mathrm{~J}$ \\
\hline 18 & $4 W$ & 0 & 0 & 21 & $10 E$ & 63,765 & $54,58 \pi$ \\
\hline 18 & $6 \mathrm{~W}$ & 12,894 & 11,037 & 21 & $11 \mathrm{E}$ & 109,542 & $93,76 \%$ \\
\hline 18 & $7 W$ & 0 & 0 & 21 & $12 E$ & 0 & n \\
\hline 18 & $8 W$ & 89.007 & 76,190 & 21 & $14 E$ & 0 & c \\
\hline 18 & 9w & 75,593 & 64,707 & & & & \\
\hline 18 & 3E & 218 & 186 & & & & \\
\hline 18 & $4 E$ & 35,424 & 30,322 & \multirow{3}{*}{\multicolumn{4}{|c|}{$\begin{array}{l}\text { Townships with no pumpage are listed because they have irrigation wells } \\
\text { without reported pumpage. }\end{array}$}} \\
\hline 18 & $5 E$ & 206,801 & 177,021 & & & & \\
\hline 18 & $6 E$ & 9,290 & 7,952 & & & & \\
\hline
\end{tabular}


Table 2. Distribution of 1984 ground-water pumpage for irrigation in Wisconsin, by township and range-Continued

\begin{tabular}{|c|c|c|c|}
\hline $\begin{array}{l}\text { Town- } \\
\text { ship' }\end{array}$ & Range & $\begin{array}{c}\text { Reported pumpage } \\
\text { (1000's of } \\
\text { gallons) }\end{array}$ & $\begin{array}{c}\text { Adjusted pumpage } \\
\text { (1000's of } \\
\text { gallons) }\end{array}$ \\
\hline 21 & $16 \mathrm{E}$ & 8,212 & 7.029 \\
\hline 21 & $17 \mathrm{E}$ & 541 & 463 \\
\hline 21 & $22 E$ & 125 & 107 \\
\hline 22 & $4 W$ & 0 & 0 \\
\hline 22 & $6 \mathrm{~W}$ & 0 & 0 \\
\hline 22 & $8 \mathrm{w}$ & 8,025 & 6,869 \\
\hline 22 & $6 E$ & 3,468 & 2,968 \\
\hline 22 & $7 \mathrm{E}$ & $1,120,593$ & 959,227 \\
\hline 22 & $8 \mathrm{E}$ & 407,443 & 348,771 \\
\hline 22 & $9 E$ & 433,452 & 371,034 \\
\hline 22 & $10 E$ & 74,952 & 64,158 \\
\hline 22 & $11 \mathrm{E}$ & 55,745 & 47,717 \\
\hline 22 & $12 \mathrm{E}$ & 0 & 0 \\
\hline 22 & $18 \mathrm{E}$ & 2,892 & 2,475 \\
\hline 22 & $19 E$ & 0 & 0 \\
\hline 23 & low & 0 & 0 \\
\hline 23 & $13 W$ & 0 & 0 \\
\hline 23 & $14 W$ & 0 & 0 \\
\hline 23 & $15 \mathrm{~W}$ & 0 & 0 \\
\hline 23 & $7 E$ & 286,336 & 245,103 \\
\hline 23 & $8 E$ & 749,485 & 641,559 \\
\hline 23 & $9 \mathrm{E}$ & 276,790 & 236,932 \\
\hline 23 & $10 E$ & 83,702 & 71,648 \\
\hline 23 & $11 E$ & 2,400 & 2,054 \\
\hline 23 & $12 \mathrm{E}$ & 9,360 & 8,012 \\
\hline 23 & $21 E$ & 0 & 0 \\
\hline 24 & $4 W$ & 0 & 0 \\
\hline 24 & $6 W$ & 0 & 0 \\
\hline 24 & $7 W$ & 0 & 0 \\
\hline 24 & $8 W$ & 0 & 0 \\
\hline 24 & $9 w$ & 10 & 8 \\
\hline 24 & 10w & 0 & 0 \\
\hline 24 & $13 W$ & 30,882 & 26.435 \\
\hline 24 & $18 \mathrm{~W}$ & 0 & 00.80 \\
\hline 24 & $8 \mathrm{E}$ & 77,256 & 66,131 \\
\hline 24 & $9 \mathrm{E}$ & 372,245 & 318,641 \\
\hline 24 & $10 E$ & 3,168 & 2,711 \\
\hline 24 & $11 \mathrm{E}$ & 47,000 & 40,232 \\
\hline 24 & $12 E$ & 15,577 & 13,333 \\
\hline 24 & $16 \mathrm{E}$ & 0 & 0 \\
\hline 24 & $18 \mathrm{E}$ & 360 & 308 \\
\hline 24 & $19 \mathrm{E}$ & 4,020 & 3,441 \\
\hline 24 & $20 \mathrm{E}$ & 0 & 0 \\
\hline 24 & $22 \mathrm{E}$ & 732 & 626 \\
\hline 24 & 23E & 7.020 & 6,009 \\
\hline 25 & $5 W$ & 4,200 & 3,595 \\
\hline 25 & $8 W$ & 0 & 0 \\
\hline 25 & $13 W$ & 8,364 & 7,159 \\
\hline 25 & $14 W$ & 4,228 & 3,619 \\
\hline 25 & $17 W$ & 0 & 0 \\
\hline 25 & $8 E$ & 79,590 & 68,129 \\
\hline 25 & $9 \mathrm{E}$ & 103,894 & 88,933 \\
\hline 25 & $10 E$ & 29,865 & 25,564 \\
\hline 25 & $11 E$ & 0 & 0 \\
\hline 25 & $15 E$ & 0 & 0 \\
\hline 25 & $16 \mathrm{E}$ & 210 & 179 \\
\hline 25 & $19 E$ & 0 & 0 \\
\hline 26 & $7 W$ & 0 & 0 \\
\hline 26 & $9 \mathrm{~W}$ & 0 & 0 \\
\hline 26 & 10w & 13,320 & 11,401 \\
\hline
\end{tabular}

\begin{tabular}{|c|c|c|c|}
\hline $\begin{array}{l}\text { Town- } \\
\text { ship' }\end{array}$ & Range & $\begin{array}{l}\text { Reported pumpage } \\
\text { (1000's of } \\
\text { gallons) }\end{array}$ & $\begin{array}{l}\text { Adjusted pumpage } \\
\text { (1000's of } \\
\text { gallons) }\end{array}$ \\
\hline 26 & $11 \mathrm{~W}$ & 46,432 & 39,745 \\
\hline 26 & $12 W$ & 104,625 & 89,559 \\
\hline 26 & $13 W$ & 0 & 0 \\
\hline 26 & $14 W$ & 23,760 & 20,338 \\
\hline 26 & $6 E$ & 5,856 & 5,012 \\
\hline 26 & $9 \mathrm{E}$ & 0 & 0 \\
\hline 26 & $10 E$ & 0 & 0 \\
\hline 27 & $8 W$ & 6,372 & 5,454 \\
\hline 27 & $9 \mathrm{~W}$ & 12,864 & 11,011 \\
\hline 27 & $10 W$ & 0 & 0 \\
\hline 27 & $11 \mathrm{~W}$ & 374,876 & 320,893 \\
\hline 27 & $12 W$ & 104,400 & 89,366 \\
\hline 27 & $18 W$ & 8,597 & 7,359 \\
\hline 27 & $19 w$ & 0 & 0 \\
\hline 27 & $20 \mathrm{~W}$ & 0 & 0 \\
\hline 27 & $6 E$ & 8,400 & 7,190 \\
\hline 27 & $7 \mathrm{E}$ & 17,640 & 15,099 \\
\hline 27 & $9 \mathrm{E}$ & 0 & 0 \\
\hline 27 & $10 E$ & 0 & 0 \\
\hline 27 & $11 E$ & 0 & 0 \\
\hline 27 & $20 \mathrm{E}$ & 828 & 708 \\
\hline 27 & $26 \mathrm{E}$ & 9,960 & 8,525 \\
\hline 28 & $8 W$ & 0 & 0 \\
\hline 26 & $9 \mathrm{~W}$ & 720 & 616 \\
\hline 28 & 10w & 1,332 & 1,140 \\
\hline 28 & $11 w$ & 18,945 & 16,216 \\
\hline 26 & $12 W$ & 31,528 & 26,988 \\
\hline 28 & $17 W$ & 9108 & 7796 \\
\hline 28 & $18 W$ & 300 & 256 \\
\hline 28 & $19 w$ & 0 & 0 \\
\hline 28 & $20 \mathrm{~W}$ & 0 & 0 \\
\hline 28 & $10 \mathrm{E}$ & 0 & 0 \\
\hline 28 & $18 E$ & 0 & 0 \\
\hline 28 & $19 E$ & 24,240 & 20.749 \\
\hline 28 & $26 E$ & 412 & 352 \\
\hline 29 & $8 W$ & 18,993 & 16,258 \\
\hline 29 & $9 w$ & 1,075 & 920 \\
\hline 29 & $11 W$ & 0 & 0 \\
\hline 29 & $12 W$ & 66,040 & 56,530 \\
\hline 29 & $15 W$ & 0 & 0 \\
\hline 29 & $16 \mathrm{~W}$ & 0 & 0 \\
\hline 29 & $17 \mathrm{~W}$ & 1,900 & 1,626 \\
\hline 29 & $18 W$ & 20,414 & 17,474 \\
\hline 29 & $19 \mathrm{~W}$ & 11,697 & 10,012 \\
\hline 29 & $11 E$ & 0 & 0 \\
\hline 29 & $17 \mathrm{E}$ & 0 & 0 \\
\hline 29 & $18 E$ & 68,832 & 58,920 \\
\hline 30 & $7 W$ & 0 & 0 \\
\hline 30 & $8 W$ & 12,900 & 11,042 \\
\hline 30 & $9 w$ & 4,277 & 3,661 \\
\hline 30 & $12 W$ & 0 & 0 \\
\hline 30 & $13 \omega$ & 11,745 & 10,053 \\
\hline 30 & $14 \mathrm{~W}$ & 8,035 & 6,878 \\
\hline 30 & $17 W$ & 3,000 & 2,568 \\
\hline 30 & $18 \mathrm{~W}$ & 6,048 & 5,177 \\
\hline
\end{tabular}


Table 2. Distribution of 1984 ground-water pumpage for irrigation in Wisconsin, by township and range-Continued

\begin{tabular}{|c|c|c|c|}
\hline $\begin{array}{l}\text { Town- } \\
\text { ship }^{1}\end{array}$ & Range & $\begin{array}{l}\text { Reported pumpage } \\
\text { (1000's of } \\
\text { gallons) }\end{array}$ & $\begin{array}{l}\text { Adjusted pumpage } \\
\text { (1000's of } \\
\text { gallons) }\end{array}$ \\
\hline 30 & $19 W$ & 0 & 0 \\
\hline 30 & $10 \mathrm{E}$ & 4,500 & 3,852 \\
\hline 30 & $11 E$ & 36,796 & 31,497 \\
\hline 30 & $12 \mathrm{E}$ & 2,220 & 1,900 \\
\hline 30 & $18 E$ & 0 & 0 \\
\hline 30 & $19 \mathrm{E}$ & 0 & 0 \\
\hline 31 & $10 \mathrm{w}$ & 5,280 & 4.519 \\
\hline 31 & $11 \mathrm{~W}$ & 14,700 & 12,583 \\
\hline 31 & $12 W$ & 0 & 0 \\
\hline 31 & $13 \mathrm{~W}$ & 0 & 0 \\
\hline 31 & $15 \mathrm{~W}$ & 0 & 0 \\
\hline 31 & $16 \mathrm{~W}$ & 0 & 0 \\
\hline 31 & $17 \mathrm{~W}$ & 48,103 & 41,176 \\
\hline 31 & $18 \mathrm{~W}$ & 3,580 & 3,064 \\
\hline 31 & $10 \mathrm{E}$ & 17,641 & 15,100 \\
\hline 31 & $11 \mathrm{E}$ & 73,754 & 63,133 \\
\hline 31 & $12 E$ & 0 & 0 \\
\hline 31 & $13 \mathrm{E}$ & 8,892 & 7,611 \\
\hline 31 & $14 \mathrm{E}$ & 8,169 & 6,992 \\
\hline 31 & $18 \mathrm{E}$ & 202 & 172 \\
\hline 31 & 2OE & 0 & 0 \\
\hline 31 & $28 \mathrm{E}$ & 714 & 611 \\
\hline 32 & $9 \mathrm{~W}$ & 11,610 & 9,938 \\
\hline 32 & 10w & 13,122 & 11,232 \\
\hline 32 & $11 W$ & 0 & 0 \\
\hline 32 & $12 \mathrm{~W}$ & 6,672 & 5,711 \\
\hline 32 & $13 W$ & 0 & 0 \\
\hline 32 & $15 \mathrm{~W}$ & 0 & 0 \\
\hline 32 & $17 \mathrm{~W}$ & 0 & 0 \\
\hline 32 & $18 \mathrm{~W}$ & 0 & 0 \\
\hline 32 & $19 w$ & 0 & 0 \\
\hline 32 & $8 \mathrm{E}$ & 0 & 0 \\
\hline 32 & $10 E$ & 0 & 0 \\
\hline 32 & $11 \mathrm{E}$ & 86,544 & 74,081 \\
\hline 32 & $12 \mathrm{E}$ & 48,100 & 41,173 \\
\hline 32 & $14 \mathrm{E}$ & 11,400 & 9,758 \\
\hline 32 & $15 \mathrm{E}$ & 8,100 & 6,933 \\
\hline 32 & $19 \mathrm{E}$ & 293 & 250 \\
\hline 32 & $20 \mathrm{E}$ & 12,960 & 11.093 \\
\hline 33 & $7 w$ & 0 & 0 \\
\hline 33 & 9w & 32,580 & 27,888 \\
\hline 33 & $10 \mathrm{~W}$ & 127,555 & 109,187 \\
\hline 33 & $11 \mathrm{~W}$ & 201,096 & 172,138 \\
\hline 33 & $14 \mathrm{~W}$ & 0 & 0 \\
\hline 33 & $15 \mathrm{~W}$ & 0 & 0 \\
\hline
\end{tabular}

\begin{tabular}{|c|c|c|c|}
\hline $\begin{array}{l}\text { Town- } \\
\text { ship' }\end{array}$ & Range & $\begin{array}{c}\text { Reported pumpage } \\
\text { (1000's of } \\
\text { gallons) }\end{array}$ & $\begin{array}{l}\text { Adjusted pumpage } \\
\text { (1000's of } \\
\text { gallons) }\end{array}$ \\
\hline 33 & $18 \mathrm{~W}$ & 0 & 0 \\
\hline 33 & $19 \mathrm{~W}$ & 14,700 & 12,583 \\
\hline 33 & $10 E$ & 2,925 & 2,503 \\
\hline 33 & $30 \mathrm{E}$ & 0 & 0 \\
\hline 34 & $11 W$ & 131,813 & 112,831 \\
\hline 34 & $12 \mathrm{~W}$ & 7,626 & 6,527 \\
\hline 34 & $16 \mathrm{~W}$ & 0 & 0 \\
\hline 34 & $11 \mathrm{E}$ & 15,900 & 13,610 \\
\hline 34 & $29 \mathrm{E}$ & 0 & 0 \\
\hline 34 & $30 \mathrm{E}$ & 0 & 0 \\
\hline 35 & $5 w$ & 0 & 0 \\
\hline 35 & 10w & 0 & 0 \\
\hline 35 & $11 \mathrm{w}$ & 68,428 & 58,574 \\
\hline 35 & $13 \mathrm{~W}$ & 0 & 0 \\
\hline 35 & $18 W$ & 0 & 0 \\
\hline 35 & $6 E$ & 0 & 0 \\
\hline 35 & $7 E$ & 0 & 0 \\
\hline 35 & $12 \mathrm{E}$ & 10,140 & 8,679 \\
\hline 36 & $10 \mathrm{w}$ & 0 & 0 \\
\hline 36 & $11 w$ & 10,512 & 8,998 \\
\hline 36 & $6 E$ & 0 & 0 \\
\hline 36 & $8 \mathbf{E}$ & 0 & 0 \\
\hline 37 & $7 w$ & 3,780 & 3,235 \\
\hline 37 & $12 w$ & 9,360 & 8,012 \\
\hline 37 & $7 E$ & 0 & 0 \\
\hline 37 & $10 \mathrm{E}$ & 0 & $n$ \\
\hline 38 & $11 \mathrm{~W}$ & 9,612 & 8,227 \\
\hline 38 & $14 \mathrm{~W}$ & 0 & $n$ \\
\hline 38 & $10 \mathrm{E}$ & 0 & 0 \\
\hline 39 & $9 \mathrm{w}$ & 0 & c \\
\hline 39 & 10w & 0 & 0 \\
\hline 39 & $11 \mathrm{~W}$ & 0 & c \\
\hline 39 & $12 \mathrm{~W}$ & 7,965 & 6,818 \\
\hline 39 & $13 \mathbf{W}$ & 0 & n \\
\hline 39 & $6 E$ & 0 & 0 \\
\hline 40 & $11 \mathrm{~W}$ & 0 & c \\
\hline 40 & $13 w$ & 0 & 0 \\
\hline 40 & $10 \mathrm{E}$ & 19,368 & $16,57 \varepsilon$ \\
\hline 40 & $18 \mathrm{E}$ & 2,520 & 2,157 \\
\hline 40 & $19 \mathrm{E}$ & 3,567 & $3,05 \hat{\imath}$ \\
\hline 41 & $8 \mathrm{~W}$ & 0 & 0 \\
\hline 41 & $9 w$ & 6,984 & 5,978 \\
\hline \multirow[t]{2}{*}{41} & $8 E$ & 7,429 & 6,359 \\
\hline & TOTAL & $21,240,608$ & $18,181,956$ \\
\hline
\end{tabular}

1 Townships with no pumpage are listed because they have irrigaticn wells without reported pumpage.

NOTE: Figures may not add to totals because of independent rornding. 
Table 3. Distribution of 1984 ground-water pumpage for irrigation in Wisconsin, by county

\begin{tabular}{|c|c|c|c|c|c|}
\hline County & $\begin{array}{c}1984 \\
\text { reported pumpage } \\
\text { (1000's of } \\
\text { gallons) }\end{array}$ & $\begin{array}{c}1984 \\
\text { adjusted pumpage } \\
\text { (1000's of } \\
\text { gallons) }\end{array}$ & County & $\begin{array}{c}1984 \\
\text { reported pumpage } \\
\text { (1000's of } \\
\text { gallons) }\end{array}$ & $\begin{array}{c}1984 \\
\text { adjusted pumpage } \\
(1000 \text { 's of } \\
\text { gallons) }\end{array}$ \\
\hline Adams & $2,873,676$ & $2,459,866$ & Marquette & 129,229 & 110,620 \\
\hline Ashl and & 0 & 0 & Menominee & 0 & 0 \\
\hline Barron & 566,824 & 485,201 & & & \\
\hline Bayfield & 0 & 0 & Milwaukee & 37,878 & 32,423 \\
\hline Brown & 4,752 & 4,067 & $\begin{array}{l}\text { Monroe } \\
\text { Oconto }\end{array}$ & $\begin{array}{r}6,253 \\
102,202\end{array}$ & $\begin{array}{r}5,352 \\
87,484\end{array}$ \\
\hline Buffalo & 231,780 & 198,403 & Oneida & 0 & 0 \\
\hline Burnett & 0 & 0 & Outagamie & 12,005 & 10,276 \\
\hline Calunet & 8,313 & 7,115 & & & \\
\hline Chippewa & 56,187 & 48,096 & Ozaukee & 6,126 & 5,243 \\
\hline Clark & 0 & 0 & $\begin{array}{l}\text { Pepin } \\
\text { Pierce }\end{array}$ & $\begin{array}{r}12,592 \\
8,597\end{array}$ & $\begin{array}{r}10,778 \\
7,359\end{array}$ \\
\hline Columbia & 60,499 & 51,787 & Polk & 14,700 & 12,583 \\
\hline Crawf ord & 0 & 0 & Portage & $5,839,630$ & $4,998,722$ \\
\hline Dane & 257,176 & 220,142 & & & \\
\hline Dodge & 6,288 & 5,382 & Price & 0 & 0 \\
\hline Door & 11,086 & 9,489 & $\begin{array}{l}\text { Racine } \\
\text { Richland }\end{array}$ & $\begin{array}{l}54,045 \\
80,689\end{array}$ & $\begin{array}{l}46,262 \\
69,069\end{array}$ \\
\hline Douglas & 0 & 0 & Rock & 358,273 & 306,681 \\
\hline Dunn & 805,086 & 689,153 & Rusk & 32,580 & 27,888 \\
\hline Eau Claire & 36,756 & 31,463 & & & \\
\hline Florence & 6,087 & 5,210 & st. Croix & 104,150 & 89,152 \\
\hline \multirow[t]{2}{*}{ Fond du Lac } & 15,654 & 13,399 & Sauk & 833,393 & 713,384 \\
\hline & & & Sawyer & 10,764 & 9,214 \\
\hline Forest & 10,140 & 8,679 & Shawano & 210 & 179 \\
\hline Grant & 15,624 & 13,374 & Sheboygan & 12,811 & 10,966 \\
\hline Green & 144,171 & 123,410 & & & \\
\hline Green Lake & 40,882 & 34,995 & Taylor & 0 & 0 \\
\hline \multirow[t]{2}{*}{ I owa } & 590,110 & 505,134 & Trempealeau & 143,674 & 122,984 \\
\hline & & & Vernon & 0 & 0 \\
\hline Iron & 0 & 0 & Vilas & 26,797 & 22,938 \\
\hline Jackson & 80 & 68 & Walworth & 15,945 & 13,648 \\
\hline Jefferson & 96,234 & 82,376 & Washburn & 26,937 & 23,058 \\
\hline Juneau & $1,036,074$ & 886,879 & & & \\
\hline \multirow[t]{2}{*}{ Kenosha } & 19,818 & 16,964 & Washington & 0 & 0 \\
\hline & & & Waukesha & 237,910 & 203,650 \\
\hline Kewaunee & 7,020 & 6,009 & Waupaca & 239,624 & 205,118 \\
\hline La Crosse & 41,900 & 35,866 & Waushara & $5,284,499$ & $4,523,530$ \\
\hline Laf ayette & 0 & 0 & Winnebago & $\begin{array}{r}5,876 \\
\end{array}$ & 5,029 \\
\hline Langl ade & 323,861 & 277.224 & Wood & 281,407 & 240,884 \\
\hline Lincoln & 0 & 0 & & & \\
\hline Manit towoc & 6,085 & 5,208 & & & \\
\hline Marathon & 36,396 & 31,155 & & & \\
\hline Marinette & 13,253 & 11,344 & Total & $21,240,608$ & $18,181,956$ \\
\hline
\end{tabular}

1 Zero pumpage indicated that no pumpage was reported.

NOTE: Figures may not add to totals because of independent rounding. 